\section{Ciao Sandro, ora nel cielo brilla una stella in più ...}

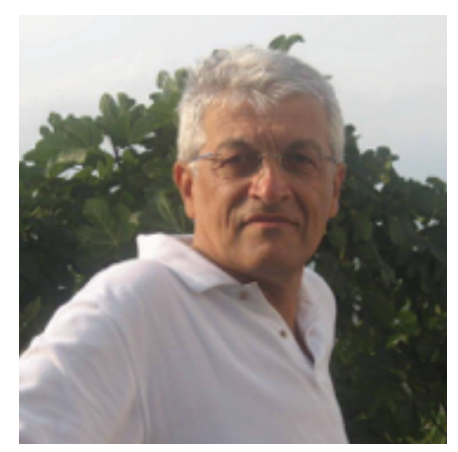

Ciao Sandro,

ricordare un amico, un collega che ha contribuito a scrivere la storia della nefrologia italiana rattrista profondamente. I ricordi, belli o brutti che siano, non li potremo più rivivere con te, caro Sandro, che hai fatto parte della nostra vita, prima come Presidente dell'Associazione EDTNA/ERCA Filiale

Italiana e poi con il tuo supporto, di spalla, nel comitato scientifico SIAN. Sono i ricordi, indelebili nella nostra mente, a tenerci uniti e rappresentano un ponte tra noi e te, tra la terra e la vita eterna, un mondo che possiamo solo immaginare e in cui tu Sandro, ora, sei giunto con serenità.

Quando scriviamo per un amico e un collega che ha fatto parte della nostra vita, lo facciamo consci che il tempo trascorso e le traversie vissute sono impressi nella nostra memoria. Ascoltando i nostri sentimenti la penna scorre sul foglio al ritmo delle nostre emozioni.

La nostra avventura ha avuto inizio al termine degli anni '80, inizi anni ' 90 . Via via che partecipavamo ai congressi europei EDTNA/ERCA capivamo la ricchezza interiore che ogni evento lasciava dentro noi, accrescendoci professionalmente. Sentivamo l'esigenza di trasmettere le conoscenze ai colleghi creando una società scientifica italiana.

Allora esisteva solo l'Associazione Infermieristica Nefrologica EDTNA/ERCA europea. Molti di noi facevano parte di quel mondo associativo e ci sentimmo travolti dal turbinio e dal desiderio di creare qualcosa per il territorio nazionale italiano. Aurelia Castagnoli, Marisa Pegoraro, Gabriella Cerruti, Anna Cotto, Margherita Rivetti, Valentina Paris e la sottoscritta riuscirono nel 1991 a realizzare questa impresa, dando vita a EDTNA/ERCA Associazione Europea, Filiale Italiana. Da quel momento ci siamo impegnati costantemente per promuovere, accrescere e far emergere le competenze e la professionalità infermieristica in area nefrologica.

Received: January 3, 2021

Accepted: January 14, 2021

Published online: February 18, 2021

Indirizzo per la corrispondenza:

Maria Pia Zito

Via Montanara 8/3

40050 Castenaso, Bologna - Italy

mariapiazito1@gmail.com
Molti colleghi si sono succeduti nel corso degli anni, ti hanno conosciuto, ammirato, apprezzato e oggi sono tutti qui a ricordarti con affetto: Laura, Patrizia, Francesco, Simona, Ada, Cristina, Mara, Cinzia e molti altri.

Sandro sei stato il nostro presidente EDTNA/ERCA Filiale Italiana da quel lontano 1992 fino al 2008. Negli anni della tua presidenza hai sempre creduto e sostenuto la mission associativa, hai realizzato e condiviso molte attività con l'obiettivo di far emergere l'associazione. Ricordo bene le battaglie per il riconoscimento di un gruppo coeso e compatto di infermieri nefrologici dell'associazione unica europea ed italiana. Hai scritto, pubblicato, insegnato ai professionisti infermieri della nefrologia come esercitare e divulgare l'assistenza infermieristica in dialisi.

Hai rappresentato EDTNA/ERCA Filiale Italiana ed Europea per decenni. Oggi, molti infermieri ti sono grati per avere elevato il loro livello professionale. Quanti convegni di nefrologia, giornate formative costruiti insieme e quanta esperienza mettevamo in campo ogni volta per arricchire la cultura infermieristica.

Grazie Sandro. Grazie per aver condiviso insieme a noi un tratto della tua vita, per aver contribuito alla crescita della nostra professione infermieristica, per la tua cultura, il tuo spessore, la tua umanità e il tuo essere "sempre" un Signore.

Nel 2019 ti abbiamo voluto come relatore al congresso nazionale della nuova società scientifica SIAN per narrare la storia della nefrologia infermieristica. Non poteva che essere descritta da te!

Ci hai donato una sferzata di vitalità, spronandoci ad emergere come professione e come professionisti, dimostrando la tua grandezza e lo straordinario "infermiere" che rimarrà indelebile per sempre nella storia della nefrologia italiana.

Lasci un vuoto incolmabile. Non ti dimenticheremo mai. Tutta la società SIAN-Italia si unisce al dolore della famiglia per la grave perdita.

Concludo con questa breve poesia di Fernando Pessoa " $L a$ morte è la curva della strada"

... Un amico è per sempre e tu per me lo sei ancora, anzi oggi più che mai: oggi che non usciamo più assieme, oggi che non ascolti più le mie lamentele al telefono, oggi che le nostre vite sono cambiate e tu chissà dove sei e chissà cosa stai facendo. Sarai per sempre mio amico perché una parte di te sarà sempre con me ed è proprio questo che mi fa sperare che andrà tutto meglio, che un giorno ci ritroveremo e che io, nel frattempo, dovrò fare tutto quello che abbiamo fatto insieme finora: sorridere e vivere.

Ciao Sandro, ora nel cielo brilla una stella in più ...

\author{
Maria Pia Zito \\ Onorata di averti conosciuto e aver lavorato con te \\ Si unisce tutto il Direttivo SIAN-Italia
}




\section{Ricordi}
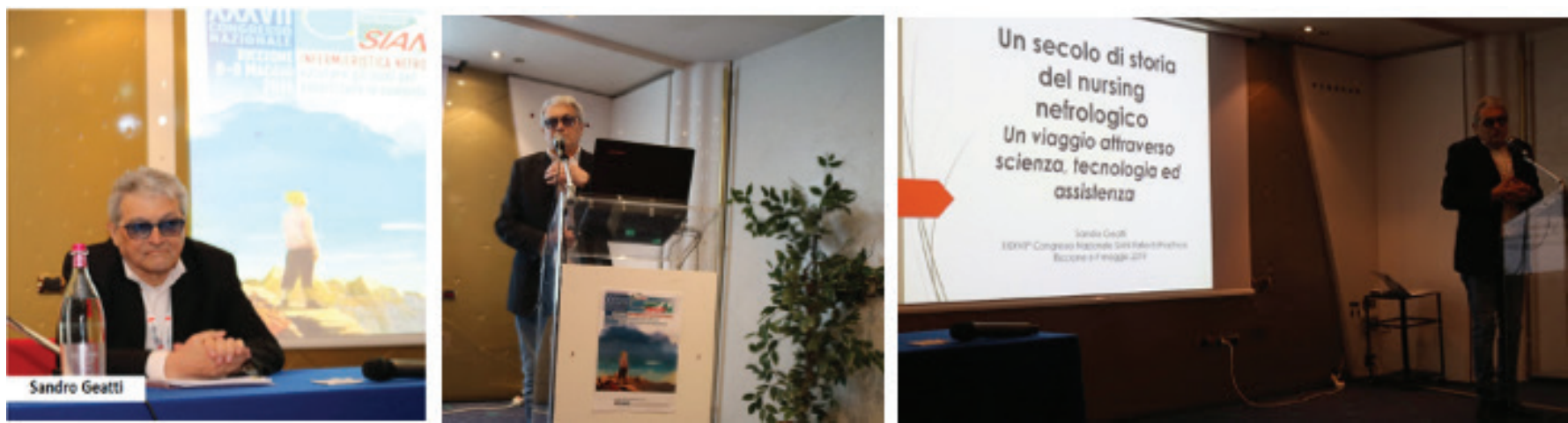

Congresso Nazionale (2019)
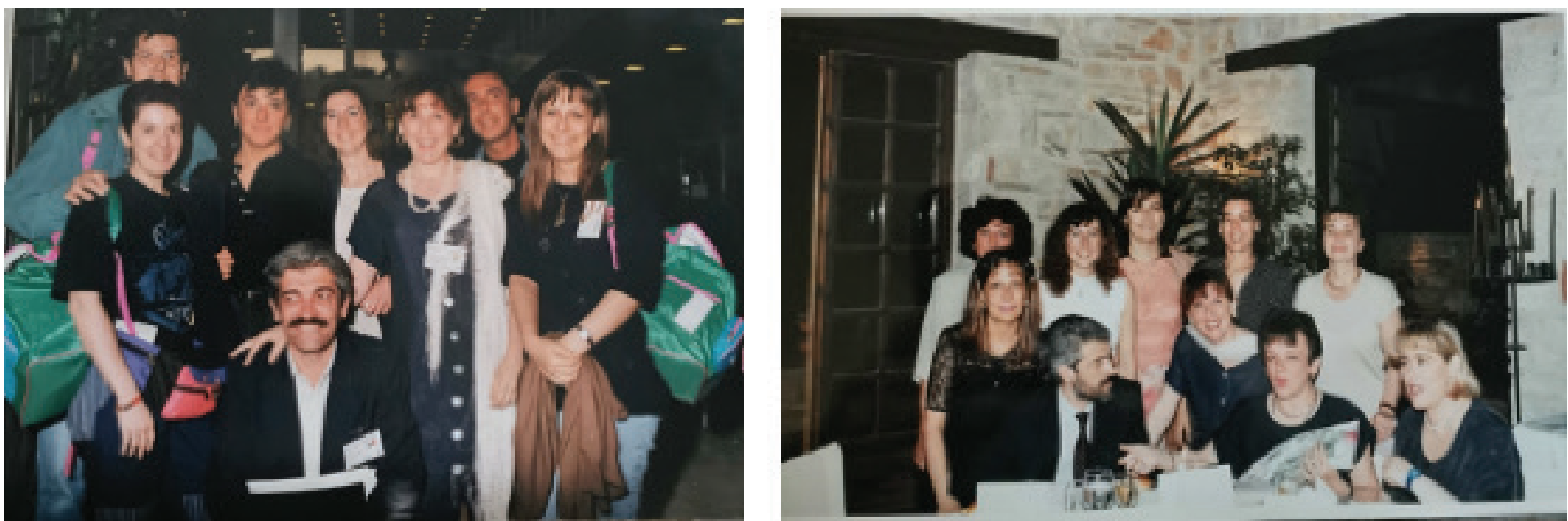

Atene (1995)
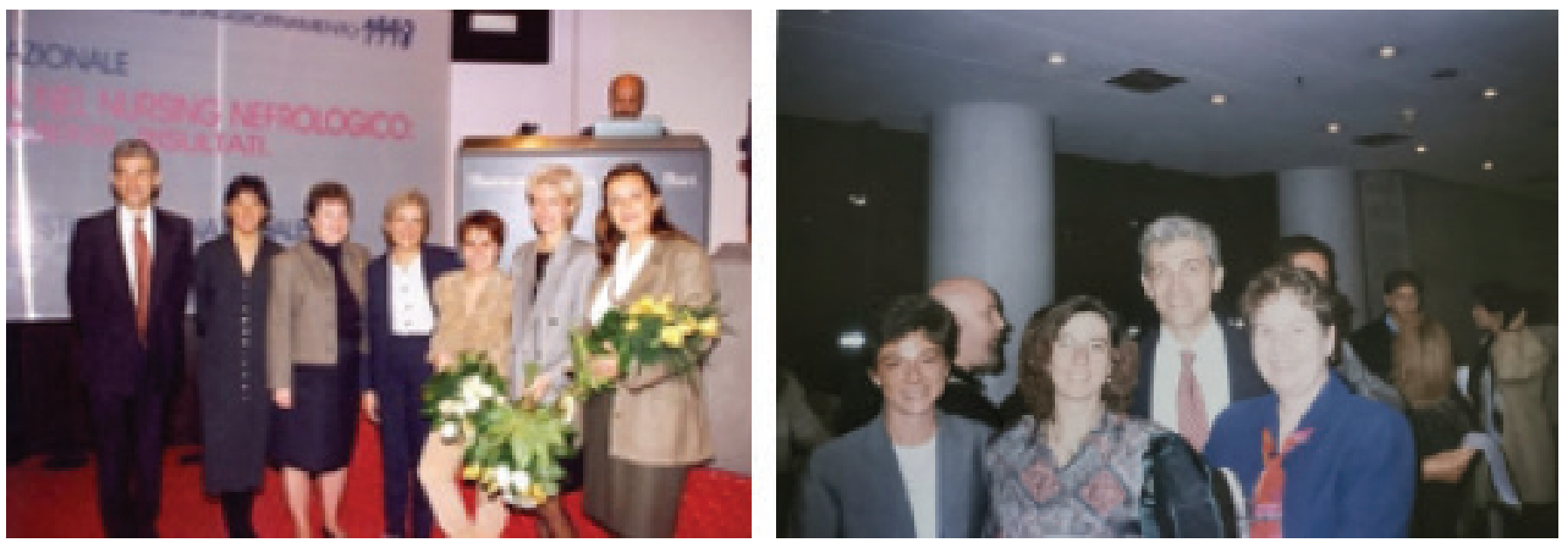

Bari (1998) 

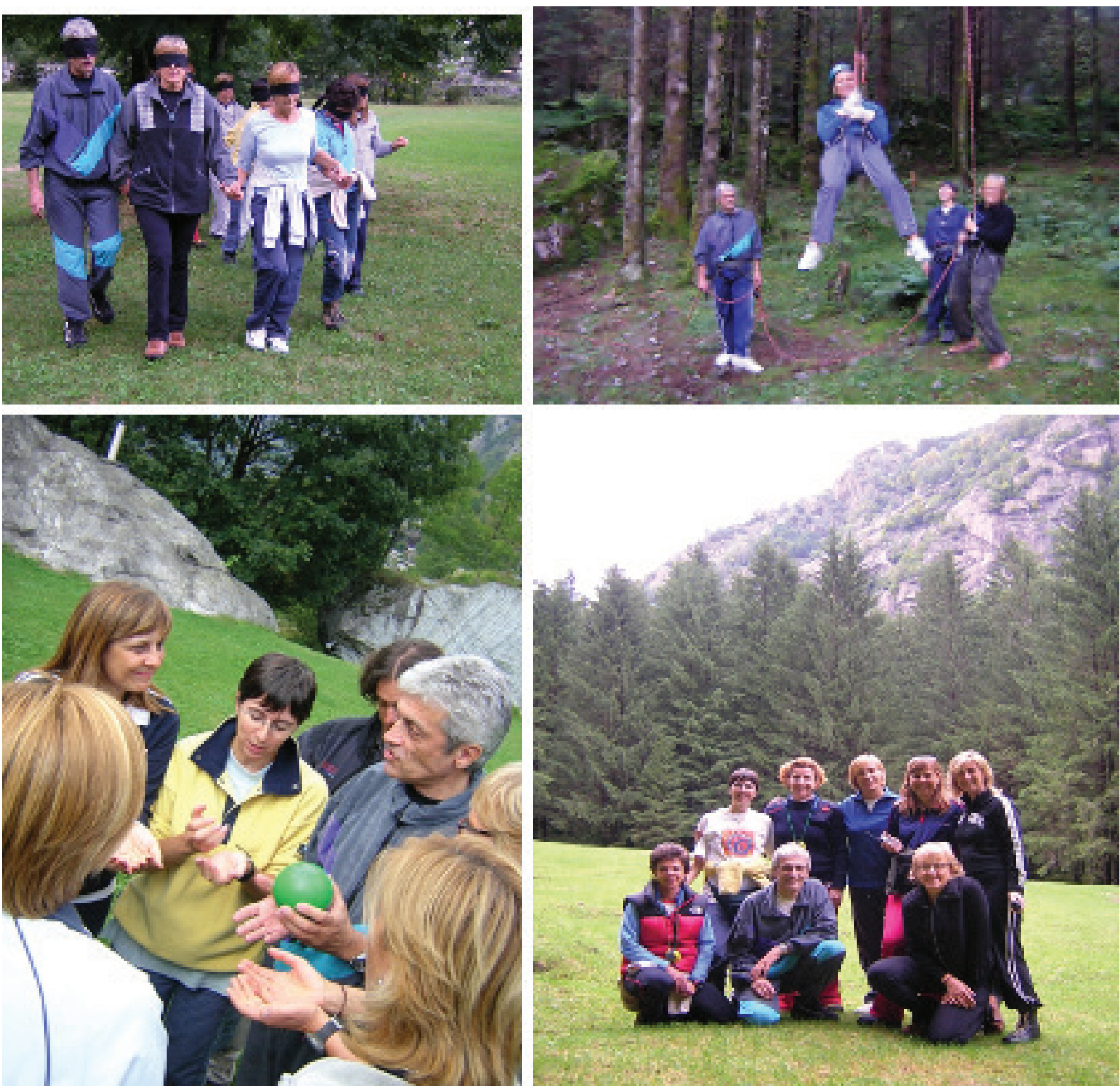

II nostro corso di sopravvivenza (2004). Ci ha aiutato ad essere una squadra .... 